\title{
Epidemiological Research in Physical Activity and Sedentary Behaviors
}

\author{
Wonwoo Byun $\mathbb{D}^{1},{ }^{1}$ Jung-Min Lee, ${ }^{2}$ Yang Bai, ${ }^{3}$ and Youngdeok Kim ${ }^{4}{ }^{4}$ \\ ${ }^{1}$ Department of Health, Kinesiology, and Recreation, University of Utah, Salt Lake City, UT, USA \\ ${ }^{2}$ School of Physical Education, Kyung Hee University, Yongin, Republic of Korea \\ ${ }^{3}$ Department of Rehabilitation and Movement Science, University of Vermont, Burlington, VT, USA \\ ${ }^{4}$ Department of Kinesiology and Sport Management, Texas Tech University, Lubbock, TX, USA
}

Correspondence should be addressed to Wonwoo Byun; won.byun@utah.edu

Received 28 October 2018; Accepted 28 October 2018; Published 28 November 2018

Copyright (C) 2018 Wonwoo Byun et al. This is an open access article distributed under the Creative Commons Attribution License, which permits unrestricted use, distribution, and reproduction in any medium, provided the original work is properly cited.

Accumulating data from population-based epidemiological studies is needed to demonstrate positive health outcomes associated with regular physical activity across lifespans. It becomes clearer that physical activity plays a critical role in preventing a number of chronic diseases, such as cardiovascular disease [1], type 2 diabetes [2], obesity [3], osteoporosis [4], and cancer [5], as well as premature mortality [6, 7]. To date, unfortunately, only a small proportion of the population in developed and/or developing countries is physically active enough to gain the associated health benefits. Thus it places physical inactivity (i.e., lack of physical activity) as one of the major public health concerns worldwide.

Further, more recently, there is growing evidence concerning sedentary behavior, independent of physical inactivity, as an emerging health risk behavior in contemporary societies. Recent studies showed that not only the total volume of time spent being sedentary, but also the manner in which sedentary time is accumulated are associated with health outcomes $[6,8]$. However, available data are limited and sometimes inconsistent, leaving a gap in understanding the role of sedentary behavior on health in various population groups. As part of continuous efforts to extend our understanding in two of the most influencing lifestyle factors on human health, physical activity, and sedentary behaviors, this special issue focuses on a broad range of topics in epidemiological research on physical activity and sedentary behavior within a behavioral epidemiology framework.

We invited investigators to submit original research articles as well as review articles addressing recent advances in epidemiological studies defining physical activity and/or sedentary behavior as either an exposure or an outcome variable. More specifically, this special issue is dedicated (1) to understanding how physical activity and/or sedentary behavior independently and/or jointly influence the risk of developing adverse health outcomes and longevity in various population groups, (2) to exploring the factors at various levels (e.g., individual, environmental) influencing physical activity and sedentary behavior, (3) to improving physical activity and sedentary behavior assessments in epidemiological research; and (4) to exploring the evidence-based intervention strategies to modify the behaviors at various population groups.

Given the significant burden and the increasing prevalence of chronic disease, the social significance of physical activity and sedentary behavior have never been greater. This special issue has strived to include, in a comprehensive manner, novel research employing methodologies in epidemiology studies such as randomized controlled trials, community-based interventions, observational study, and systematic reviews. With the recognition of difficulties in translating scientific knowledge generated from research into public health practice, we believe that the knowledge provided by this special issue would further contribute to generating high-quality evidence on the health benefits of physical activity and promoting physical activity in practical settings.

\section{Conflicts of Interest}

The authors declare that there are no conflicts of interest regarding the publication of this paper. 


\section{Acknowledgments}

The authors of this editorial wish to thank external reviewers and all authors of articles published in this special issue.

\section{Wonwoo Byun \\ Jung-Min Lee \\ Yang Bai \\ Youngdeok Kim}

\section{References}

[1] S. N. BlaiR, "Physical inactivity and cardiovascular disease risk in women," Medicine \& Science in Sports \& Exercise, vol. 28, no. 1, pp. 9-10, 1996.

[2] D. Aune, T. Norat, M. Leitzmann, S. Tonstad, and L. J. Vatten, "Physical activity and the risk of type 2 diabetes: a systematic review and dose-response meta-analysis," European Journal of Epidemiology, vol. 30, no. 7, pp. 529-542, 2015.

[3] T. Moholdt, U. Wisløff, S. Lydersen, and J. Nauman, "Current physical activity guidelines for health are insufficient to mitigate long-term weight gain: More data in the fitness versus fatness debate (The HUNT study, Norway)," British Journal of Sports Medicine, vol. 48, no. 20, pp. 1489-1496, 2014.

[4] W.-C. Li, Y.-C. Chen, R.-S. Yang, and J.-Y. Tsauo, "Effects of exercise programmes on quality of life in osteoporotic and osteopenic postmenopausal women: A systematic review and meta-analysis," Clinical Rehabilitation, vol. 23, no. 10, pp. 888896, 2009.

[5] L. Liu, Y. Shi, T. Li et al., "Leisure time physical activity and cancer risk: evaluation of the WHO's recommendation based on 126 high-quality epidemiological studies," British Journal of Sports Medicine, vol. 50, no. 6, pp. 372-378, 2016.

[6] U. Ekelund, J. Steene-Johannessen, W. J. Brown et al., "Does physical activity attenuate, or even eliminate, the detrimental association of sitting time with mortality? A harmonised metaanalysis of data from more than 1 million men and women," The Lancet, vol. 388, no. 10051, pp. 1302-1310, 2016.

[7] S. C. I. Moore, A. V. Patel, C. E. Matthews et al., "Leisure time physical activity of moderate to vigorous intensity and mortality: a large pooled cohort analysis," PLoS Medicine, vol. 9, no. 11, Article ID e1001335, 2012.

[8] C. E. Matthews, S. K. Keadle, R. P. Troiano et al., "Accelerometermeasured dose-response for physical activity, sedentary time, and mortality in US adults," American Journal of Clinical Nutrition, vol. 104, no. 5, pp. 1424-1432, 2016. 


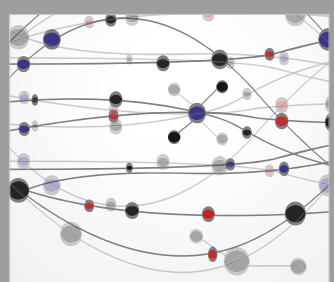

The Scientific World Journal
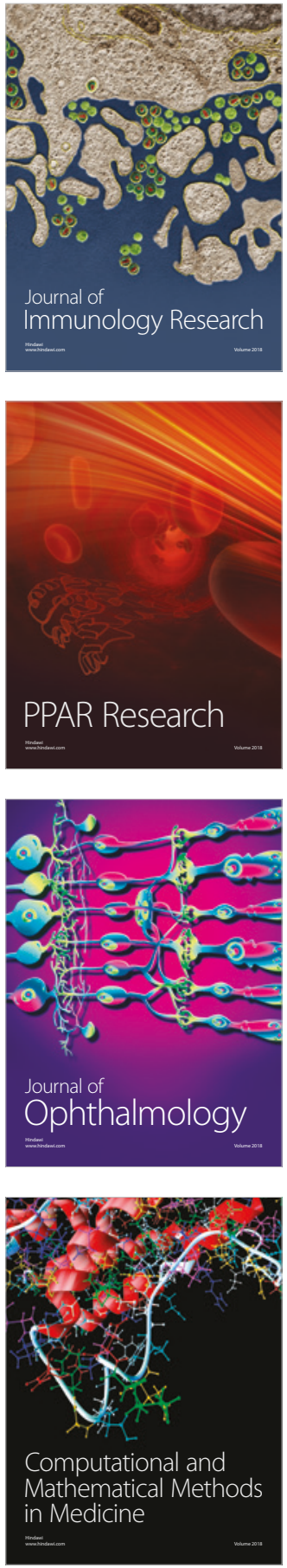

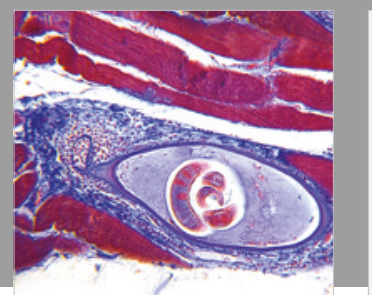

Gastroenterology Research and Practice

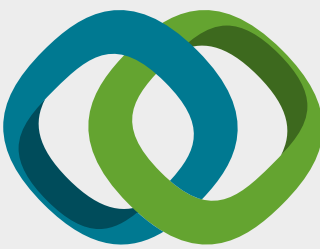

\section{Hindawi}

Submit your manuscripts at

www.hindawi.com
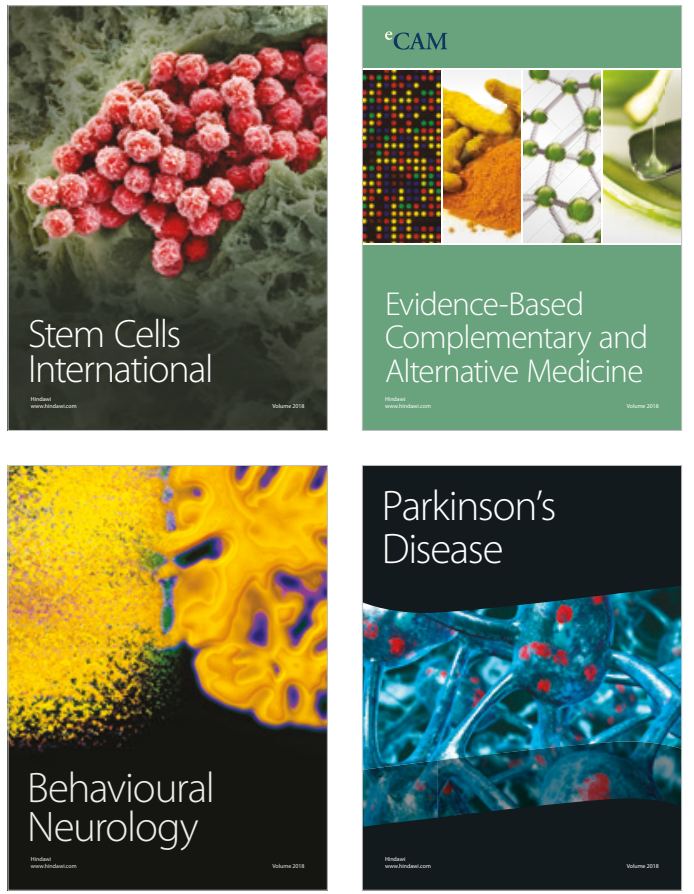

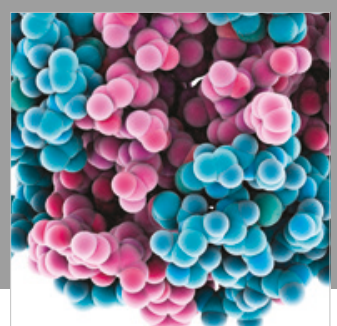

ournal of

Diabetes Research

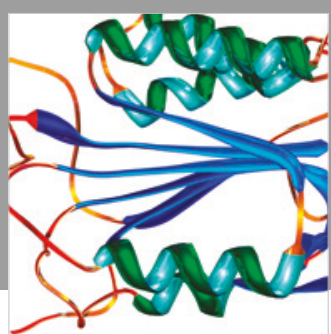

Disease Markers
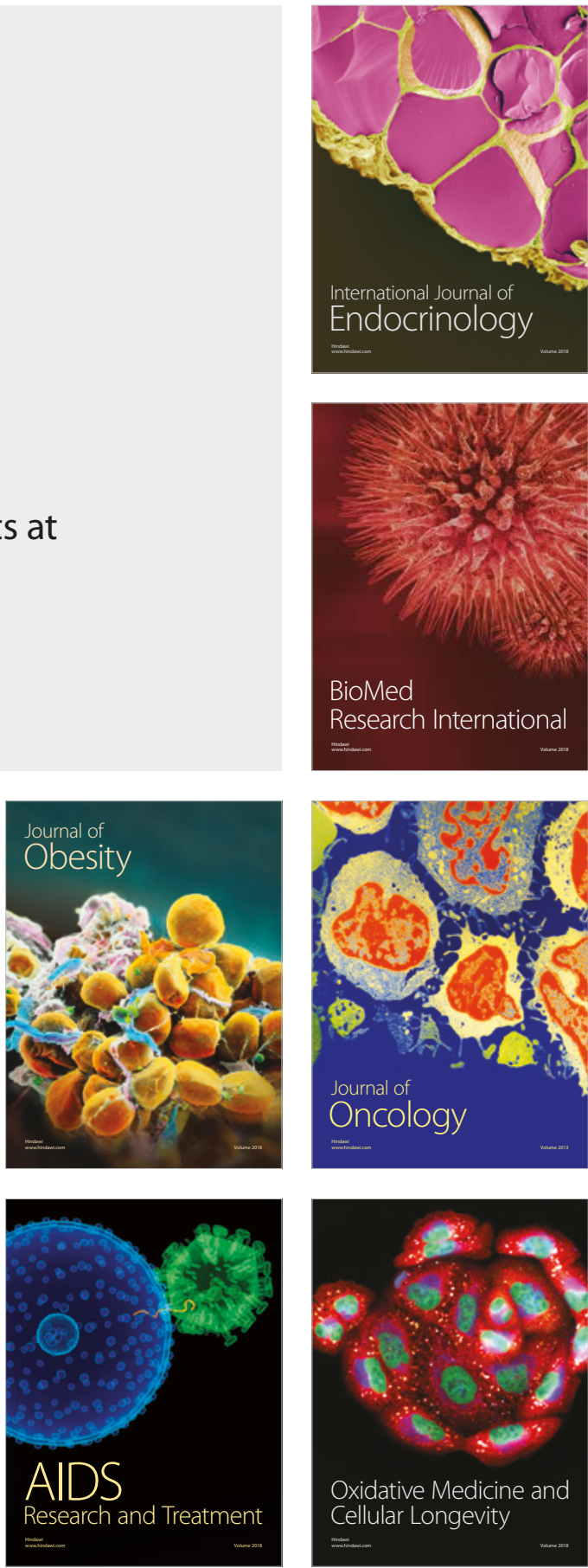